\title{
Correction to: The Effects of Lifestyle and/or Vitamin D Supplementation Interventions on Pregnancy Outcomes: What Have we Learned from the DALI Studies?
}

\author{
Jürgen Harreiter ${ }^{1}$ - Gernot Desoye ${ }^{2}$ - Mireille N. M. van Poppel ${ }^{3}$ - Alexandra Kautzky-Willer ${ }^{1} \cdot$ Fidelma Dunne $^{4}$. \\ Rosa Corcoy ${ }^{5,6,7}$. Roland Devlieger ${ }^{8}$. David Simmons ${ }^{9,10}$. On behalf of the DALI Consortium • Juan M. Adelantado . \\ Peter Damm • Elizabeth Reinhardt Mathiesen - Dorte Moeller Jensen - Lise Lotte T. Anderson • Annunziata Lapolla • \\ Maria G. Dalfrà • Alessandra Bertolotto • Ewa Wender-Ozegowska • Agnieszka Zawiejska • David J. Hill • Frank J. Snoek
}

(C) Springer Science+Business Media, LLC, part of Springer Nature 2020

Correction to: Current Diabetes Reports (2019) 19: 162 https://doi.org/10.1007/s11892-019-1282-7

The original version of this review article unfortunately contained a mistake. The ISRCTN number reflected in "DALI Study Design" and "Compliance with Ethical Standards" sections was incorrect. It should be "ISRCTN70595832" instead of "ISRCTN7059583".

Publisher's Note Springer Nature remains neutral with regard to jurisdictional claims in published maps and institutional affiliations.

The online version of the original article can be found at https://doi.org/ $10.1007 / \mathrm{s} 11892-019-1282-7$

David Simmons

da.simmons@westernsydney.edu.au

Jürgen Harreiter

juergen.harreiter@meduniwien.ac.at

Gernot Desoye

gernot.desoye@medunigraz.at

Mireille N. M. van Poppel

mireille.van-poppel@uni-graz.at

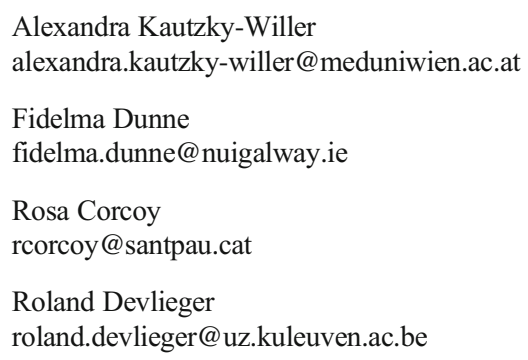

Roland Devlieger

roland.devlieger@uz.kuleuven.ac.be

Extended author information available on the last page of the article 


\section{Affiliations}

Jürgen Harreiter ${ }^{1} \cdot$ Gernot Desoye $^{2} \cdot$ Mireille N. M. van Poppel ${ }^{3} \cdot$ Alexandra Kautzky-Willer $^{1} \cdot$ Fidelma Dunne $^{4}$. Rosa Corcoy ${ }^{5,6,7} \cdot$ Roland Devlieger $^{8} \cdot$ David Simmons $^{9,10}$. Juan M. Adelantado • Peter Damm •

Elizabeth Reinhardt Mathiesen - Dorte Moeller Jensen - Lise Lotte T. Anderson • Annunziata Lapolla • Maria G. Dalfrà • Alessandra Bertolotto - Ewa Wender-Ozegowska - Agnieszka Zawiejska • David J. Hill • Frank J. Snoek • On behalf of the DALI Consortium

1 Gender Medicine Unit, Division of Endocrinology and Metabolism, Department of Medicine III, Medical University of Vienna,

Vienna, Austria

2 Department of Obstetrics and Gynecology, Medical University of Graz, Graz, Austria

3 Institute of Sport Science, University of Graz, Graz, Austria

4 National University of Ireland, Galway, Ireland

5 Institut de Recerca de l'Hospital de la Santa Creu i Sant Pau, Barcelona, Spain

6 CIBER Bioengineering, Biomaterials and Nanotechnology, Instituto de Salud Carlos III, Madrid, Spain
7 Departament de Medicina, Universitat Autònoma de Barcelona, Bellaterra, Spain

8 KU Leuven Department of Development and Regeneration: Pregnancy, Fetus and Neonate, Gynaecology and Obstetrics, University Hospitals Leuven, Leuven, Belgium

9 Institute of Metabolic Science, Addenbrookes Hospital, Cambridge, England

10 School of Medicine, Macarthur Clinical School, Western Sydney University, Locked Bag 1797, Campbelltown, NSW 2751, Australia 